\title{
Application of the DSDM methodology and the Django framework for the patient registration system of the Alfonso Villagómez pediatric hospital emergency service
}

\section{Aplicación de la metodología DSDM y del framework Django para el sistema de registro de pacientes del servicio de emergencia del hospital pediátrico Alfonso Villagómez}

Corresponding Author:

Blanca Hidalgo

blanca.hidalgo@espoch.edu.ec

Received: 24 December 2019

Accepted: 2 January 2020

Published: 8 January 2020

Publishing services provided by Knowledge E

(c) Blanca Hidalgo et al. This article is distributed under the terms of the Creative Commons Attribution License, which permits unrestricted use and redistribution provided that the original author and source are credited.

Selection and Peer-review under the responsibility of the SIIPRIN-CITEGC Conference Committee.

\section{Blanca Hidalgo1, Jonny Guaiña1, Vinicio Ramos", Piedad Yumiseba", and Christian Gutiérrez ${ }^{2}$}

${ }^{1}$ Escuela Superior Politécnica de Chimborazo,Riobamba, Ecuador

${ }^{2}$ Cooperativa Riobamba,Riobamba, Ecuador

\section{Abstract}

This work shows the development of a computerized system for registering emergency patients at Alfonso Villagómez Hospital, using the DSDM(Dynamic Systems Development Method) agile development methodology in which 150 requirements expressed in UML(Unified Modeling language) diagrams were obtained for greater understanding; The PyCharm integrated development environment, the Django frameworks under the language Python and Bootstrap were used for the design of the templates and database management system PostgreSQL. The architecture applied was MTV(Model Template View) and once the system was implemented, the usability of software was evaluated according to the ISO/IEC 9126 standard, using the survey called USE (utility, satisfaction and ease of use), for the hypothesis the test was applied Chi square and it is concluded that the computer system is useful and meets the needs of users.

Resumen. Este trabajo muestra el desarrollo de un sistema informático del registro de pacientes de emergencia del Hospital Alfonso Villagómez, utilizando la metodología de desarrollo ágil DSDM(Metodología de Desarrollo de Sistemas Dinámicos) en el cual se obtuvieron 150 requerimientos expresados en diagramas UML(lenguaje unificado de modelado) para mayor comprensión; para el desarrollo del sistema se utilizó el entorno de desarrollo integrado PyCharm, los frameworks Django bajo el lenguaje Python y Bootstrap para el diseño de plantillas y como gestor de base de datos PostgreSQL. La arquitectura aplicada fue MTV(Modelo, Vista, Plantilla) y una vez implantado el sistema se evaluó la usabilidad de software según el estándar ISO/IEC 9126, mediante la encuesta denominada USE (utilidad, satisfacción y facilidad de uso), para la prueba de la hipótesis se aplicó la prueba de chi cuadrado y se concluye que el sistema informático es útil y satisface las necesidades de los usuarios. 
Keywords: Technology and engineering sciences, software engineering, PYTHON, DJANGO, Dynamic systems development methodology.

Palabras clave: Tecnología y ciencias de la ingeniería, ingeniería de software, PYTHON, DJANGO, Metodología de desarrollo de sistemas dinámicos.

\section{Introducción}

El hospital pediátrico “Alfonso Villagómez Román", pertenece al nivel 3 de la zona 3 del ministerio de Salud Pública del Ecuador, se encuentra ubicado en la provincia de Chimborazo cantón Riobamba. Según los autores (Guamán Coello \& Ruiz Salazar, 2017, pp. 3-5) y como tal ofrece los siguientes servicios: consulta externa, hospitalización, emergencia, enfermería, odontología, laboratorio clínico, farmacia, pediatría, cirugía, rayos $x$, epidemiología, hematología, terapia y rehabilitación física. La unidad de salud procura el desarrollo comunitario y velar por la salud de la población infantil. El registro de los pacientes atendidos en el área de emergencia, se realiza de manera manual, inicia desde la llegada del paciente al área de enfermería y la toma de información correspondiente en el formulario 008 de emergencia establecido por el Ministerio de Salud Pública; lo que provoca en muchas ocasiones inconsistencias en los diferentes reportes mensuales generados; con respecto a errores en los datos ingresados del paciente.

La solución informática desarrollada es un sistema web con la capacidad de adaptarse a los diferentes dispositivos digitales como computadores, tablets y teléfonos inteligentes, gracias a que se optó por el desarrollo del Front-end con el framework de diseño web Bootstrap 4.1.0; lo que ha permitido que el sistema se adapte en función del tamaño de la pantalla del dispositivo, además de la facilidad.

En este caso específico se escogió el framework Django el cual está desarrollado bajo el lenguaje Python; cuya filosofía es el de fomentar la reutilización del código evitando la creación de bloques de código iguales; por lo que se ha seleccionado como IDE de desarrollo a PyCharm puesto que integra el frameworks web Django y como gestor de base de datos, se utilizó PostgreSQL.

Por ser un producto de software es vital seleccionar y adoptar un método de desarrollo de software, en este caso se utilizo DSDM(Metodología de Desarrollo de Sistemas Dinámicos) sobre todo porque proporciona un ciclo de vida iterativo en el que mediante la fragmentación del proyecto en periodos cortos de tiempo permite la definición de los 
entregables correspondientes; además, tiene claramente definidos los roles y funciones del personal involucrado en el desarrollo del proyecto que tuvo una duración de 4 meses que se corresponden al mes de abril a agosto del año 2018.

En la sección 2 se describe la parte técnica relacionada al framework de desarrollo Django, el entorno de desarrollo integrado (IDE) PyCharm, el gestor de base de datos PostgreSQL y la norma de evaluación ISO/IEC 9126. En la sección 3 se presenta el proceso necesario para la implementación de la metodología DSDM en el desarrollo del sistema web, conformado por la fase del pre-proyecto, la fase del ciclo de vida del proyecto y la fase de post-proyecto; en la sección 4 se discuten los resultados del estudio y finalmente en la sección 5 se presentan las conclusiones.

\section{Antecedentes}

\subsection{Python}

Según la publicación realizada por Python Software Foundation se define a Python como un lenguaje de programación poderoso y fácil de aprender; que cuenta con las estructuras de datos eficientes y de alto nivel así con un enfoque simple pero efectivo a la programación orientada a objetos; es un lenguaje ideal para scripting y desarrollo rápido de aplicaciones en diversas áreas y sobre la mayoría de las plataformas [1]. Python es un lenguaje de programación; que es utilizado en varios campos de la tecnología, al ser atractivo para varios programadores porque permite reducir el número de líneas de código al realizar tareas básicas[2].

\subsubsection{Principios del lenguaje de programación Python}

El lenguaje de programación Python se basa en varios principios de diseño al escribir código[2], como se puede observar en la Fig. 1[2].

\subsection{Framework de desarrollo web}

Se define a un framework como una estructura software que facilita y agiliza el desarrollo de una aplicación web; se lo puede considerar también como una aplicación genérica incompleta y configurable a la que se le puede agregar complementos adicionales para construir una aplicación web concreta con un diseño reutilizable[3]. 


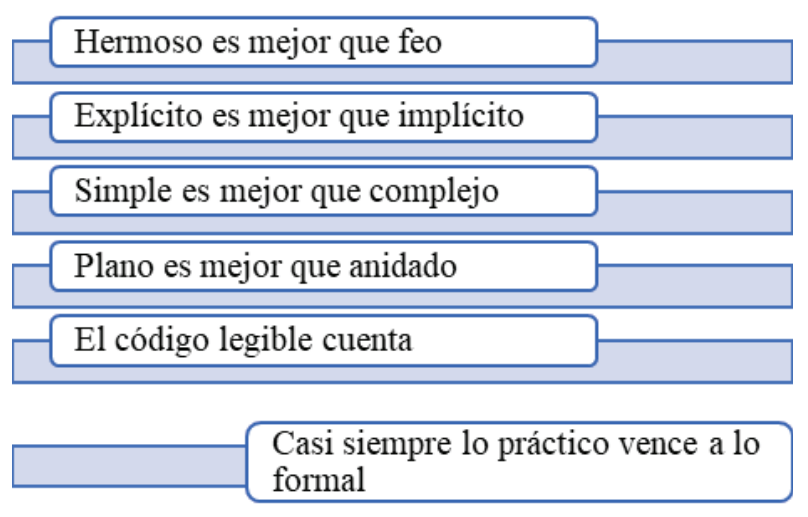

Figure 1: Principios del lenguaje de programación Python.

\subsubsection{Django}

El Framework Django es considerado con un framework web de alto nivel, muy popular y funcional, tiene como características principales el ser un framework de código abierto, escrito bajo el lenguaje de programación Python[4]. Además, Django es considerado como parte de la tercera generación del desarrollo de aplicaciones web utilizando la arquitectura MTV(Modelo, vista, plantilla) que es una variación de la conocida arquitectura MVC(Modelo, vista, controlador)[5]. Es recomendable utilizar Django en proyectos grandes que requieran modificaciones o extensiones futuras, puesto que al ser utilizado en proyectos pequeños se desperdiciaría en gran parte todas las prestaciones que presenta el framework de desarrollo[6].

\section{Arquitectura Django}

Al usar el framework Django es recomendable tratarlo con la arquitectura MTV la misma que consiste es una modificación a la arquitectura MVC[5, 7]. A continuación, se describe en qué consisten las partes de la arquitectura MTV.

\section{El modelo}

El modelo se considera como la fuente única y definitiva de los datos que posee la aplicación desarrollada, y que permite ejecutar las operaciones[8]. En la arquitectura MTV se establece que el modelo se encuentra en forma de clases, por lo que en Python se puede indicar y controlar el comportamiento de los datos almacenados [7].

\section{La vista}

La vista es considera como el elemento de la aplicación que contiene la lógica de negocio [4]; necesaria para devolver una respuesta hacia el cliente que la solicita, también procesa las peticiones o solicitudes que accederán al modelo para poder 
entregar u obtener los datos [9]. La sección de vista del modelo MTV en Django se presenta en forma de clases y de funciones en Python, en el que se determinan los datos que serán visualizados entre otras cosas, además, permite escribir código Python en lugar de instrucciones SQL(Structured Query Language) para realizar las consultas que se requieran en la vista; determinando que la vista no se responsabiliza del estilo para presentar los datos sino se encarga de los datos en si[7].

\section{La plantilla}

En la plantilla se debe de decidir la forma en la que se presentarán los datos devueltos por la vista en el navegador web; utilizando estilos CSS o brindando dinamismo a través de JavaScript [8]. Es importante recalcar que las etiquetas utilizadas por Django, permiten que sea flexible para los diseñadores del front-end, estas estructuras son limitadas para evitar un desorden poniendo cualquier tipo de código escrito en Python; con el objetivo de que lógica del sistema siga permaneciendo en la vista[7].

\section{Configuración de las rutas}

Cabe señalar que el framework Django posee un mapeo mediante direcciones URLs, que permite controlar el despliegue de las vistas, esta configuración es conocida como URLConf, que está constituido con expresiones regulares en Python. El trabajo del URLConf es leer la dirección URL del usuario que solicitó el proceso, encontrar la vista apropiada para la solicitud y pasar cualquier variable que la vista necesite para completar el proceso; además URLConf permite que las rutas que maneja Django sean agradables y entendibles para el usuario[7], este proceso se lo puede observar en la

Fig. 2.

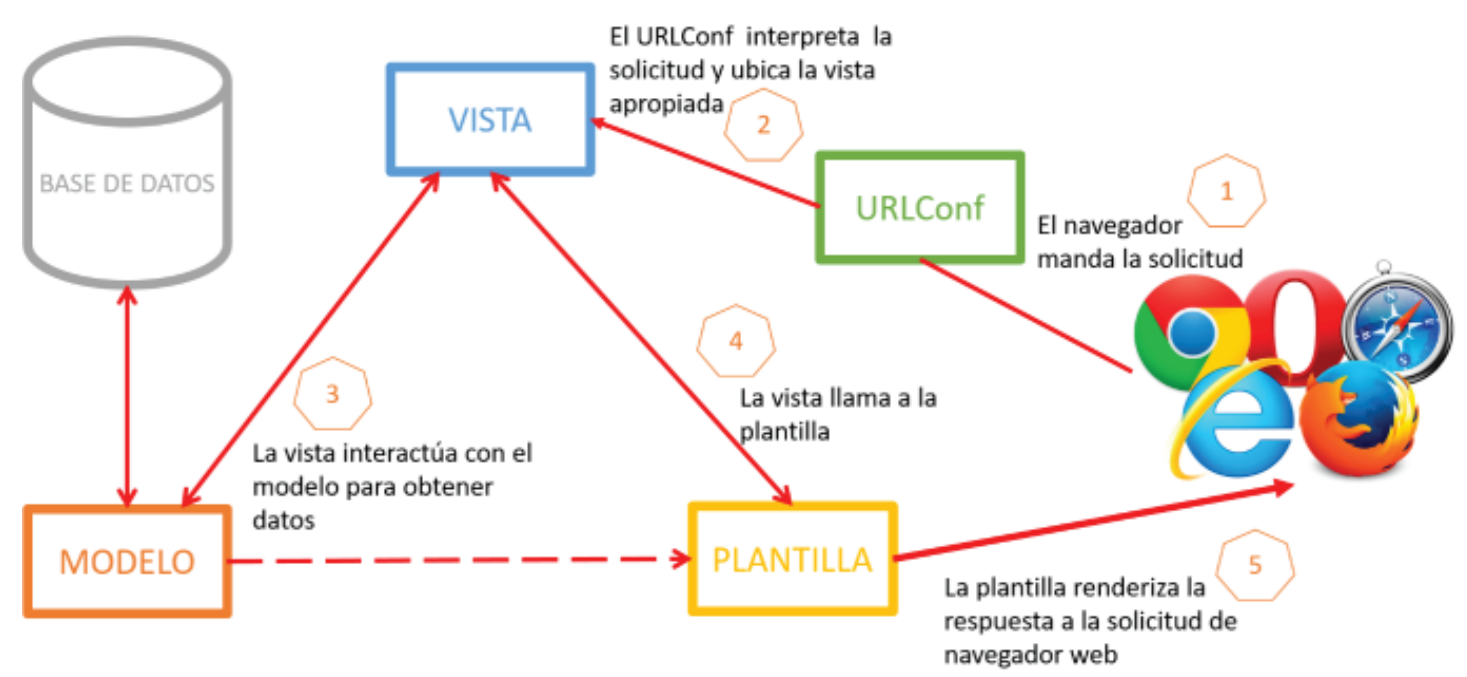

Figure 2: Configuración de las rutas de la arquitectura de Django. 


\subsubsection{Bootstrap}

Bootstrap es considerado como un framework mediante el cual se pueden crear interfaces web con estilos Css3 y JavaScript, haciendo que se adapten de manera automática al tamaño del dispositivo desde el que se acceda a la interfaz, por lo que puede ser utilizado en el desarrollo de cualquier proyecto. Bootstrap es un entorno de trabajo robusto en relación a otros frameworks debido a la gran comunidad de desarrolladores en Git Hub que brindan el soporte correspondiente [10].

\subsection{Sistema de gestión de base de datos PostgreSQL}

PostgreSQL es considerado como un SGBD(sistema de gestión de bases de datos) relacional orientada a objetos que posee un conjunto de funcionalidades avanzadas por lo que se sitúa al mismo nivel de varios SGBD comerciales; se distribuye bajo la licencia BSD que permite su uso, modificación y redistribución sin afectar el copyright del software a sus autores originales [11].

\subsection{Norma de evaluación ISO/IEC 9126}

La norma Internacional ISO/IEC 9126 fue publicada en 1992, es utilizada para la evaluación de la calidad de software desde diferentes criterios [12]. Esta norma se divide en calidad interna, externa y en uso, como se muestra a continuación en la Tabla 1.

TABLE 1: Características de calidad según la norma ISO/IEC 9126.

$\begin{array}{ll}\text { TIPO DE CALIDAD } & \text { CARACTERÍSTICAS A EVALUAR } \\ \text { Interna y Externa } & \text { - Funcionalidad } \\ & \text { - Fiabilidad / Confiabilidad } \\ & \text { - Usabilidad } \\ & \text { - Eficiencia } \\ & \text { - Mantenibilidad } \\ & \text { - Portabilidad } \\ \text { En uso } & \text { - Eficacia } \\ & \text { - Productividad } \\ & \text { - Satisfacción } \\ & \text { - Seguridad }\end{array}$

\subsubsection{Usabilidad}

Se define a la usabilidad como la capacidad del software de ser entendido, aprendido, y usado en forma fácil y atractiva. Esta característica es determinada por los usuarios 
finales y los usuarios indirectos del software [12]. La característica de usabilidad se divide en 5 criterios como se puede observar en la Fig. 3.

\begin{tabular}{ll}
\hline U & Entendimiento \\
S & A \\
B & Operabilidad \\
I & Conformidad de uso \\
L & C \\
D & Aprendizaje \\
A & Atracción \\
D &
\end{tabular}

Figure 3: Características de la usabilidad.

\subsection{Metodología ágil de desarrollo de software DSDM}

Al realizar una revisión sobre las metodologías ágiles para el desarrollo de software [13] es recomendable tratar a cada proyecto de manera independiente para lo cual se desarrolla un subconjunto de características durante un periodo de tiempo corto, en un rango de dos y seis semanas. La característica fundamental de las metodologías ágiles es la participación constante con el cliente al punto de requerir un representante de él durante el desarrollo, lo que ayudara en gran parte al proceso de retroalimentación por parte del cliente al momento de realizar las entregas de los avances planificados. Los proyectos que utilizan las metodologías ágiles son altamente colaborativos y se adaptan de mejor manera a todos los cambios que se puedan presentar durante el desarrollo de software.

La metodología DSDM proporciona un framework para el desarrollo ágil de software, apoyándose en su continua implicación constante del cliente en el desarrollo iterativo y creciente [13].

\subsubsection{Principios de DSDM}

DSDM se basa en los siguientes principios[14]:

- Involucrar al cliente es la clave para llevar un proyecto eficiente y efectivo

- El equipo del proyecto debe tener el poder para tomar decisiones que son importantes

- DSDM se centra en la entrega frecuente de productos

- El desarrollo es iterativo e incremental 
- Todos los cambios durante el desarrollo son reversibles

- Las pruebas son realizadas durante todo el ciclo vital del proyecto

- La comunicación y cooperación entre todas las partes interesadas

\subsubsection{Fases de proyecto con DSDM}

En Fig. 4, se puede observar las diferentes fases de la metodología DSDM, así como también las distintas etapas establecida para cada fase en el desarrollo de un proyecto software aplicando dicha metodología[14].

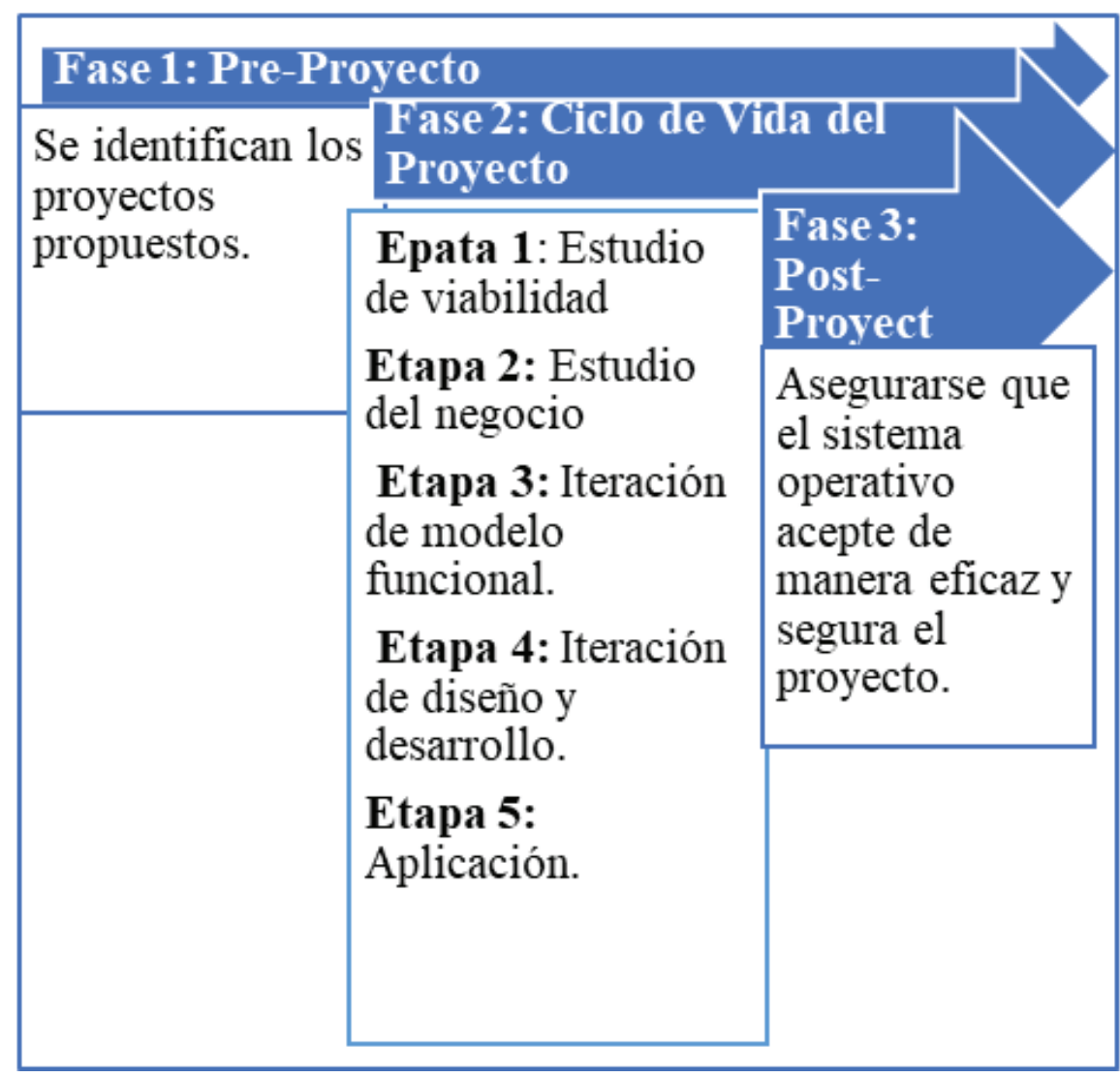

Figure 4: Fases de desarrollo establecidos por la metodología de desarrollo DSDM.

\subsubsection{Ventajas y desventajas de DSDM}

A continuación, en la Fig. 5., se pueden observar alunas de las ventajas y desventajas identificadas en la metodología DSDM en el desarrollo de un proyecto informático[15].

\section{Desarrollo del sistema informático}




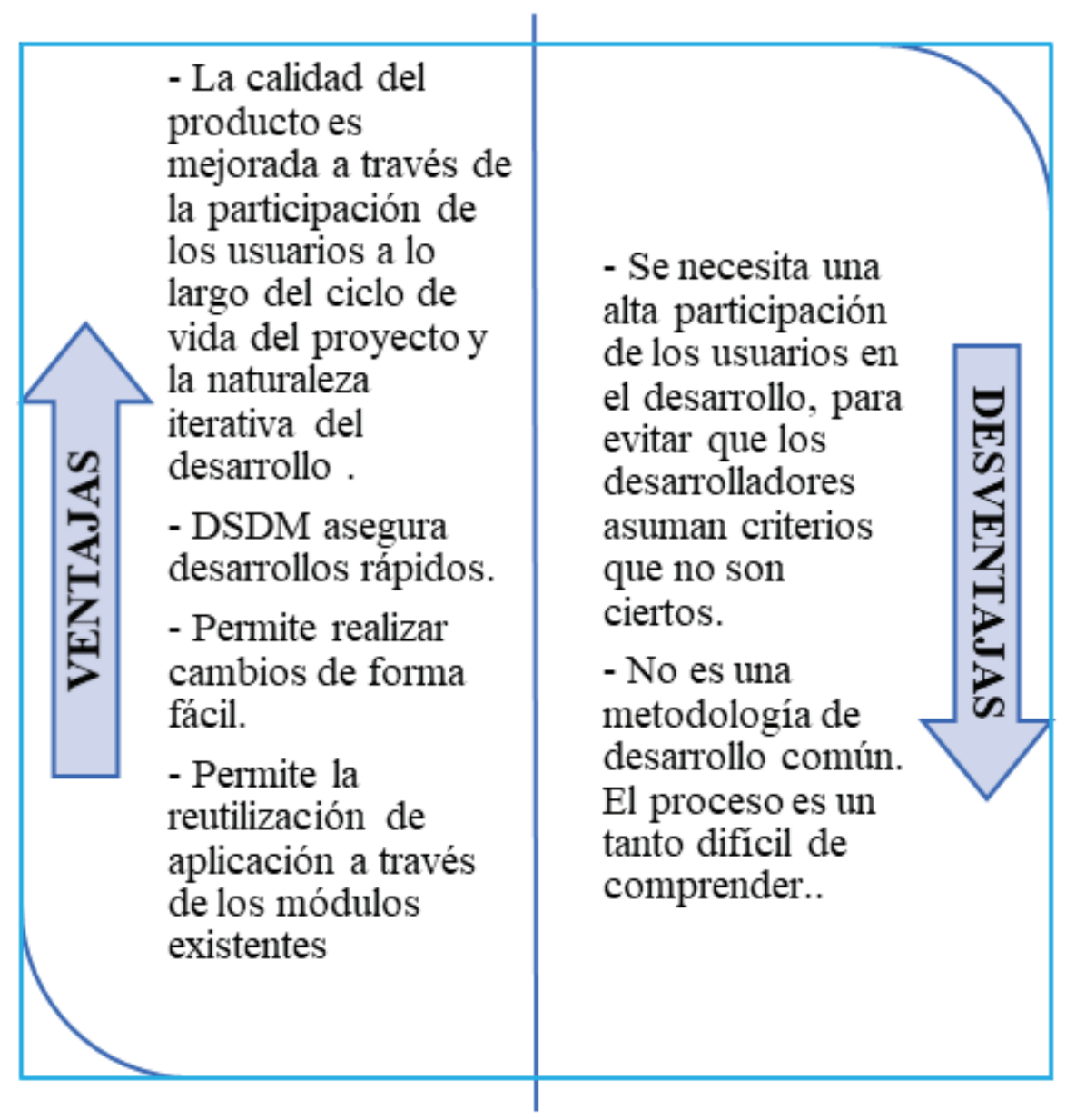

Figure 5: Ventajas y desventajas de la metodología de desarrollo DSDM.

\subsection{Pre-proyecto}

La primera fase de la metodología DSDM, consiste en identificar a las personas involucradas, y el financiamiento con el que se contó para el desarrollo del proyecto.

Los distintos roles son:

- Patrocinador ejecutivo

- Visionario

- Embajador (usuario)

- Asesor de usuario

- Jefe de proyecto

- Coordinador técnico

- Líder del equipo

- Solution developer 
- Solución tester

- Scribe

- Facilitador

\subsection{Ciclo de vida del proyecto}

Se obtuvieron 146 requerimientos funcionales divididos en varios módulos, 12 historias técnicas y 4 requerimientos no funcionales para el desarrollo del presente proyecto. De los requerimientos funcionales se realizó los diagramas UML como son los de caso de uso con sus respectivas tablas descriptivas, los diagramas de secuencia y colaboración, y los diagramas de actividades.

\subsubsection{Evaluación}

Para evaluar si el desarrollo del sistema se puede llevar a cabo se realizó el análisis y gestión de riesgos, la estimación del proyecto, el estudio de factibilidad tanto técnica, operativa y económica donde se determinó que el proyecto es factible a nivel técnico, operativo y económico se concluye que el proyecto es factible para su realización. Se estableció una solución técnica al problema de negocio, donde los recursos software para el desarrollo se eligió PyCharm ya que el sistema web está basado en el lenguaje de programación Python y pgAdmin como gestor de base de datos el cual es PostgreSQL. El software es de libre acceso y no dificulta la adquisición de estos.

\subsubsection{Estudio de la empresa}

En total se obtuvieron 12 requerimientos técnicos necesarios para el desarrollo del sistema, así como 146 funcionales. La prioridad se estableció en base a la técnica de estimación T-Shirt que mide el tamaño de las historias de usuario en base a la experiencia del desarrollador, en referencia a las tallas de ropa significando S (2 a 4 horas), M (8 horas), L (16 horas), XL (32 horas). Se tomó en cuenta también una hora de trabajo por punto estimado

Los requerimientos técnicos como los funcionales se dividieron en los siguientes módulos:

- Módulo técnico

- Módulo de servicio de emergencia 
- Módulo de atención al paciente

- Módulo de hospitalización

- Módulo de clasificación internacional de enfermedades

- Módulo de farmacia

- Módulo de usuarios

- Módulo de reportes

- Módulo de datos generales de formulario 008

- Módulo de datos generales de paciente

\subsubsection{Implementación}

La actividad de implementación fue realizada con equipo de desarrollo, con el fin de entregar el sistema funcional y realizar la capacitación al usuario, en este caso a los médicos y enfermeras del hospital.

La capacitación del sistema se realizó de tipo grupal contando con la presencia de médicos y enfermeras del área de servicio de emergencia.

\subsubsection{Evaluación de los documentos de sistema}

La documentación está realizada por cada historia de usuario e historias técnicas que evidencia su realización y funcionamiento, esta documentación incluye 298 tareas de ingeniería y 563 pruebas de aceptación, mismas que se distribuyen en la realización de 146 historias de usuario (HU) y 12 historias técnicas $(H T)$.

\subsection{Post - proyecto}

El sistema está en producción funcionando de manera eficaz y eficiente, satisfaciendo así las necesidades del servicio de emergencia del Hospital.

\section{Resultados}

En esta etapa se evaluó la calidad de software en cuanto al parámetro de usabilidad descrito en el estándar ISO/IEC 9126, mediante la aplicación de la encuesta USE (utilidad, satisfacción y facilidad de uso). 


\subsection{Evaluación de calidad de software}

Para realizar esta actividad se examinó la población y se determinó la muestra, posteriormente se analizó los datos como se describe a continuación.

\subsubsection{Población}

La población seleccionada fue de 1 administrador, 18 médicos, 13 enfermeras/auxiliares, danto un total de 32 individuos. Los cuales trabajan en el Hospital Pediátrico Alfonzo Villagómez Román.

\subsubsection{Muestra}

La muestra para el trabajo de titulación se la obtuvo aplicando el tipo de muestreo no aleatorio, del que fue seleccionado el personal que trabaja en el servicio de emergencia del hospital. Determinado de esta manera la muestra conformada por: 10 médicos, 13 enfermeras/auxiliares y un administrador, dando un total de 24 individuos para la investigación.

\subsubsection{Tabulación de resultados}

De la encuesta USE se seleccionaron 26 preguntas observadas como primordiales para evaluar la usabilidad en cuanto a 4 aspectos: utilidad, facilidad de uso, facilidad de aprendizaje y satisfacción. La encuesta aplicada cuenta con una escala Likert de 7 puntos donde 1 representa en desacuerdo y 7 de acuerdo, además cuenta con un campo N/A (no aplica). Los datos obtenidos fueron separados por secciones para el proceso de tabulación, cada sección consta de un conjunto de preguntas.

Una vez tabulados los resultados de cada una de las secciones se procedió a tabular los totales para obtener un resumen general de toda la encuesta como se muestra en la tabla 2.

La Fig. 6. Muestra el gráfico estadístico de la tabulación de los datos obtenidos de acuerdo con la escala Likert 
TABLE 2: Tabulación por secciones de la encuesta aplicada.

\begin{tabular}{|l|c|c|c|c|c|c|c|c|}
\hline & $\mathbf{1}$ & $\mathbf{2}$ & $\mathbf{3}$ & $\mathbf{4}$ & $\mathbf{5}$ & $\mathbf{6}$ & $\mathbf{7}$ & N/A \\
\hline Utilidad & 0 & 0 & 0 & 12 & 17 & 25 & 110 & 4 \\
\hline Facilidad de uso & 0 & 0 & 1 & 18 & 25 & 33 & 185 & 2 \\
\hline $\begin{array}{l}\text { Facilidad de } \\
\text { aprendizaje }\end{array}$ & 0 & 0 & 5 & 2 & 9 & 16 & 64 & 0 \\
\hline Satisfacción & 0 & 0 & 7 & 3 & 6 & 8 & 72 & 0 \\
\hline Total & 0 & 0 & 13 & 35 & 57 & 82 & 431 & 6 \\
\hline
\end{tabular}

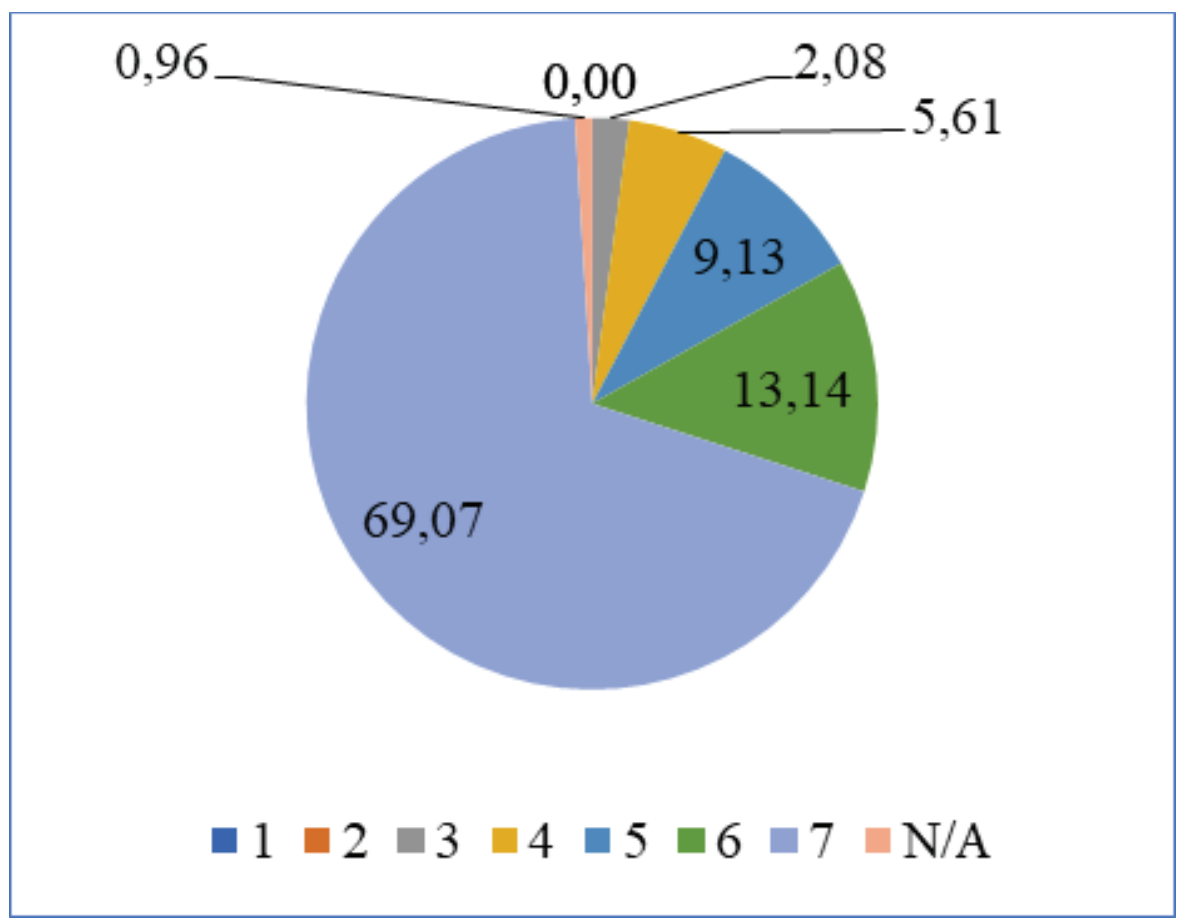

Figure 6: Resultados por secciones de la encuesta aplicada de acuerdo con la escala Likert.

\subsubsection{Análisis}

Al finalizar el análisis de la tabla 2 y la figura 6 se destaca que lo ideal era obtener un total de 624 respuestas en la opción 7 en todas las preguntas relacionadas con la evaluación en cuanto a la usabilidad del sistema. Sin embargo, se obtuvo del valor ideal esperado un $69.07 \%$ de individuos encuestados están satisfechos con el sistema web; y lo califican como: útil, fácil de usar y aprender; el 27.88\% de individuos están satisfechos al usarlo pero tienen algunos inconvenientes en su uso, aunque este criterio podría cambiar mientras dure el proceso de adaptación y familiarización con el sistema; por otro lado el $3.04 \%$ de individuos restantes tienen problemas al utilizarlo debido a que consideran que el sistema no funciona como ellos esperan por lo que no están satisfechos al usarlo lo que genera dificultades al realizar las actividades establecidas. 


\subsubsection{Diseño del experimento}

Una vez que se obtuvo los valores totales se aplicó la técnica de Chi Cuadrado para lo cual se clasificó los valores de la escala de Likert en 3 categorías: mala (N/A, 1-2-3), buena (4-5-6), y excelente (7). Además, se formuló la hipótesis nula $(\mathrm{HO})$ y la hipótesis alternativa(H1), como se detalla a continuación:

HO: Los usuarios no están de acuerdo con que el sistema web para el servicio de emergencia del Hospital Pediátrico Alfonso Villagómez es usable en cuanto a utilidad, facilidad de uso, facilidad de aprendizaje y satisfacción.

H1: Los usuarios están de acuerdo con que el sistema web para el servicio de emergencia del Hospital Pediátrico Alfonso Villagómez es usable en cuanto a utilidad, facilidad de uso, facilidad de aprendizaje y satisfacción.

Los indicadores utilizados en la prueba de la hipótesis fueron: utilidad, facilidad de uso, facilidad de aprendizaje y grado de satisfacción de los usuarios del sistema.

Se realizó la tabulación de los valores observados, y los valores esperados, y se procedió a calcular el chi cuadrado como se muestra en la tabla 3 aplicando la fórmula 1.

$$
x^{2}=\sum \frac{\left(o_{i}-e_{i}\right)^{2}}{e_{i}}
$$

TABLE 3: Cálculo de chi cuadrado.

\begin{tabular}{l|c|c|c|c|}
$\begin{array}{l}\text { VALORES } \\
\text { OBSERVA- } \\
\text { DOS } \\
\text { (O) }\end{array}$ & $\begin{array}{c}\text { VALORES } \\
\text { ESPERADOS (E) }\end{array}$ & O-E & $(\mathbf{O}-\mathbf{E})^{2}$ & $(\mathbf{O}-\mathbf{E})^{2} / \mathbf{E}$ \\
\hline 4 & 5,1154 & $-1,1154$ & 1,2441 & 0,2432 \\
\hline 3 & 8,0385 & $-5,0385$ & 25,3861 & 3,1581 \\
\hline 5 & 2,9231 & 2,0769 & 4,3136 & 1,4757 \\
\hline 7 & 2,9231 & 4,0769 & 16,6213 & 5,6862 \\
\hline 54 & 46,8462 & 7,1538 & 51,1775 & 1,0925 \\
\hline 76 & 73,6154 & 2,3846 & 5,6864 & 0,0772 \\
\hline 27 & 26,7692 & 0,2308 & 0,0533 & 0,0020 \\
\hline 17 & 26,7692 & $-9,7692$ & 95,4379 & 3,5652 \\
\hline 110 & 116,0385 & $-6,0385$ & 36,4630 & 0,3142 \\
\hline 185 & 182,3462 & 2,6538 & 7,0429 & 0,0386 \\
\hline 64 & 66,3077 & $-2,3077$ & 5,3254 & 0,0803 \\
\hline 72 & 66,3077 & 5,6923 & 32,4024 & 0,4887 \\
\hline CHI CUADRADO & & & & 16,2220 \\
\hline
\end{tabular}


Se procedió a calcular los grados de libertad para obtener chi de la tabla aplicando la fórmula 2:

$$
g l=(\text { filas }-1) *(\text { columnas }-1)
$$

Con lo que se obtiene el siguiente resultado:

$$
g l=(4-1) *(3-1)=6
$$

Se aplicó un nivel de significancia de a=5\% y se observó que el valor de chi de la tabla es de 12.592; dado que 16,2220>12.592; razón por la cual se acepta la hipótesis alternativa y se rechaza la hipótesis nula, es decir, se acepta que los usuarios están de acuerdo con que el sistema web para el servicio de emergencia del Hospital Pediátrico Alfonso Villagómez es usable en cuanto a utilidad, facilidad de uso, facilidad de aprendizaje y satisfacción, con lo cual también se asegura que no existen diferencias significativas entre las 4 características de usabilidad evaluadas.

\section{Conclusiones}

Django permitió mayor rapidez en la construcción del sistema informático, con su arquitectura MTV que aporta varias ventajas como: el que separa y organiza el código, soporta diferentes gestores de base de datos, en la que se usó PostgreSQL, para la plantilla se diseñó con Bootstrap que permitió hacer un sistema responsive que se adapta a cualquier dimensión de pantalla y dispositivos de navegación.

Se aplicó la metodología de desarrollo ágil DSDM en la que se realizaron las tres etapas (pre-proyecto, ciclo de vida de proyecto y post-proyecto); gracias a los principios bajo los cuales fue desarrollada la metodología nos permitió construir un sistema acorte a las necesidades establecidas mediante la comunicación y cooperación del equipo de desarrollo.

Se aplicó la técnica de chi cuadrado donde el resultado de 16,222 es mayor al valor crítico 12.592 que se obtiene con un nivel de significancia de a=5\% y 6 grados de libertad; lo que significa que se rechaza la hipótesis nula y se acepta la hipótesis alternativa que indica que los usuarios están de acuerdo que el sistema informático es usable.

\section{References}

[1] Python Software Foundation, «1. Introducción --- Tutorial de Python 3.6.3 documentation», Tutorial de Python. [En línea]. Disponible en: http://docs.python. 
org.ar/tutorial/3/real-index.html. [Accedido: 27-ago-2019].

[2] I. Challenger, Y. Díaz, y R. Becerra, «Theprogramming languagePython», Ciencias Holguín, pp. 1-13, 05-abr-2014.

[3] J. J. Gutiérrez, «¿Qué es un framework web?», p. 4.

[4] A. Garcia, «¿Cuáles son las principales debilidades de Python como lenguaje de programación? - Quora», Quora, 16-ene-2017. [En línea]. Disponible en: https://es.quora.com/Cu\{\%\}C3\{\%\}A1les-son-las-principales-debilidades-de-Pythoncomo-lenguaje-de-programaci\{\%\}C3\{\%\}B3n. [Accedido: 27-ago-2019].

[5] S. Infante, «Curso Django: Entendiendo como trabaja Django», Enetndiendo Django, 30-abr-2012.

[6] J. Sánchez, «Para qué proyectos usar Django | Freelancer Blog», Freelancer Blog, 26-mar-2015.

[7] J. L. Condori, «Phython - DjangoFramework de desarrollo web para perfeccionistasBasado en el Modelo MTV», Revista de Información, Tecnología y Sociedad, p. 2, nov-2012.

[8] G. VanRossum, «El tutorial de Python», oct-2017.

[9] R. Caldera, «ESTUDIO DEL FRAMEWORK DE DESARROLLO WEB DJANGO», Universidad de Alcalá, España, 2017.

[10] B. Villarreal, «Desarrollo de un sistema web para la gestión de procesos de un restaurante», UNIVERSIDAD POLITÉCNICA DE MADRID, Madrid España, 2016.

[11] R. Camps Paré y Universitat Oberta de Catalunya, Bases de datos. Barcelona: UOC, 2005.

[12] C. A. L. Garcia y E. M. Mazo, «GUIA TECNICA PARA EVALUACION DE SOFTWARE», p. 41.

[13] J. De la Cruz y B. López, «metodologia de desarrollo de sistemas dinamicos o Dynamic Systems Dev...», 12-oct-2014. [En línea]. Disponible en: https://es.slideshare. net/xomin100/dsdm-40167415. [Accedido: 27-ago-2019].

[14] «Métodos de Desarrollo de Sistemas Dinámicos(DSDM) I Ingeniería del Software UAH», 29-mar-2015. [En línea]. Disponible en: https://ingenieriadelsoftwareuah2015. wordpress.com/2015/03/29/metodos-de-desarrollo-de-sistemas-dinamicos-dsdm/. [Accedido: 27-ago-2019].

[15] C. Villamarin y R. Sosa, «Ventajas y Desventajas - utmfci». [En línea]. Disponible en: https://sites.google.com/site/utmfci/home/ventajas-y-desventajas. [Accedido: 30sep-2019]. 